\title{
Serve una borraccia
}

\section{We need a flask}

Giovanni Lodi

Dipartimento di Scienze Biomediche, Chirurgiche e Odontoiatriche

Università degli Studi di Milano

via Beldiletto 1/3 Milano 20142

Italia

giovanni.lodi@unimi.it

tel. + 390250319021

fax. +390250319041

Allora ci volle un'epidemia globale con migliaia di morti.

Fu tra la fine degli ottanta e gli inizi dei novanta che le abitudini degli odontoiatri di tutto il mondo cambiarono radicalmente a causa di una nuova letale malattia che si trasmetteva col sangue. Perché prima della comparsa dell'AIDS, guanti, mascherine, strumenti monouso e autoclavi erano l'eccezione, non la regola. Poi, per proteggere i pazienti (e se stessi), i dentisti adottarono misure oggi considerate irrinunciabili, e a distanza di 30 anni i casi di trasmissione dell'infezione in uno studio odontoiatrico si contano sulle dita di una mano e non credo siano state documentate sieroconversioni per motivi professionali.

Chissà se anche oggi siamo alla vigilia di modifiche altrettanto profonde. Perché se l'emergenza AIDS interessava tanti cittadini del mondo, quella attuale li coinvolge tutti, tutti e sette miliardi. Come avrete immaginato sto parlando dell'emergenza ambientale in tutte le sue declinazioni: dai mutamenti climatici all'inquinamento da plastica. Problemi per i quali forse abbiamo già superato il punto di non ritorno, ma che non per questo possiamo ignorare come individui e come categoria.

Alcune associazioni nazionali, come quella inglese e australiana, e la FDI World Dental Federation, hanno aperto la discussione su una pratica sostenibile, pubblicando documenti di indirizzo che elencano indicazioni di massima, senza entrare però troppo nel merito. Per esempio auspicando una odontoiatria che riduca significativamente il monouso senza però compromettere la sicurezza del paziente. Uno scenario che per diventare realtà richiederà procedure e strumenti ancora tutti da inventare.

E intanto sono già nate le prime cliniche odontoiatriche green, anche se in questo caso esiste il dubbio che il vero obiettivo sia quello promozionale.

C'è molto da lavorare, soprattutto su noi stessi e sulle nostre abitudini. leri in clinica ero seduto al grande tavolo attorno al quale passiamo insieme la pausa pranzo. Tutti i presenti, prevalentemente studenti, avevano davanti a sé delle borracce molto stilose e piene d'acqua del rubinetto (a Milano è ottima). L'unico con una bottiglietta d'acqua di plastica, di quelle da mezzo litro delle macchinette, ero io.

Buona lettura, 